\title{
MOAGEM FINA DE MINÉRIO DE COBRE DE ALTA EFICIÊNCIA ENERGÉTICA
}

\author{
M. G. BERGERMAN ${ }^{1}$, L. C. R. MACHADO², A. MIRANDA², R. FONSECA ${ }^{2}$, H. DELBONI JR. ${ }^{3}$ \\ ${ }^{1}$ Instituto de Ciência e Tecnologia - Universidade Federal de Alfenas \\ ${ }^{2}$ Departamento de Cobre - Vale S.A. \\ ${ }^{3}$ Departamento de Engenharia de Minas e de Petróleo - Universidade de São Paulo \\ mauricio.bergerman@unifal-mg.edu.br ${ }^{1}$
}

Artigo submetido em novembro/2013 e aceito em dezembro/2013

DOI: http://dx.doi.org/10.15628/holos.2014.1797

\section{RESUMO}

$\mathrm{Na}$ área de moagem secundária, ou remoagem, a preocupação com a eficiência energética se mostra de extrema importância. Em geral, os consumos específicos desta etapa são superiores aos da etapa primária. Além disso, tem se observado uma tendência de afinamento das granulometrias de moagem nos novos projetos de minerais metálicos, o que leva a cada vez uma maior necessidade de gastos nesta etapa, tanto em termos de custos de implantação como de operação. Nos novos projetos de cobre da Vale, granulometrias de remoagem da ordem de 40 a $20 \mu \mathrm{m}$ tem se mostrado necessárias. Os moinhos de bolas convencionais, adequados para moagem primária, mostram-se poucos eficientes em termos de aproveitamento de energia, quando são necessários produtos abaixo de $50 \mu \mathrm{m}$. A fim de avaliar as melhores alternativas tecnológicas disponíveis para estas aplicações de remoagem, a Vale desenvolve estudos em moinhos de carga agitada, como Vertimill ${ }^{\circledR}$. No presente trabalho serãodiscutidos os resultados de amostragens em escala industrial com o moinho Vertical da Usina do Sossego nos primeiros anos de operação do mesmo, comparando os resultados com os consumos específicos determinados em escala laboratorial.

PALAVRAS-CHAVE: cominuição, remoagem, cobre, Vertimill ${ }^{\circledR}$

\section{HIGH ENERGY EFFICIENCY COPPER ORE FINE GRINDING}

\begin{abstract}
In the areas of secondary grinding, or regrinding, the energy efficiency of the circuit is very important. Usually, the specific energy consumption of these stages is higher than at the primary stages. Besides that, there is a trend at the new projects where a finer grinding target is observed, what increases the capital and operational costs of the projects. At Vale new copper projects, grinding sizes of the concentrate around 20 to 40 ? $\mathrm{m}$ has been necessary. The ball mill, traditionally used for these
\end{abstract}

applications, shows a low energy efficiency at grinding target bellow 50 ? $\mathrm{m}$. In order to evaluate the available Technologies for the regrind of Vale coppers ores, the company is developing studies with the stirred mill technology, such as the Vertimill ${ }^{\circledR}$. The present paper will illustrate the results of several industrial surveys at the Sossego regrind circuit at the first years of operation, comparing the results with the laboratory sizing data.

KEYWORDS: comminution, regrind, copper, Vertimill ${ }^{\circledR}$ 


\section{INTRODUÇÃO}

A Mina do Sossego foi a primeira da Vale a entrar em operação no processamento de minério de cobre. O depósito foi descoberto em 1997 e compreende os corpos denominados Sossego e Sequeirinho. A mina está localizada a aproximadamente $70 \mathrm{~km}$ de Carajás, próximo à cidade de Canaã dos Carajás. A usina tem capacidade para processar $41.000 \mathrm{tpd}$, o que equivale a $15 \mathrm{Mt} / \mathrm{ano}$, lavrados a céu aberto. A reserva medida??? é da ordem de $245 \mathrm{Mt}$ com um teor médio de $0,98 \%$ de cobre e $0,28 \mathrm{~g} / \mathrm{t}$ de ouro (MINBEC, 2001).

O minério é um granito com quantidade significativa de magnetita. Valores típicos de WI variam de 17 a $20 \mathrm{kWh} / \mathrm{t}$, além de possuírem um índice de abrasão relativamente alto, de 0,5.

A operação da usina iniciou-se em abril de 2004, com uma capacidade de produção de 540.000 t/ano de concentrado, com aproximadamente $30 \%$ de cobre e $8 \mathrm{~g} / \mathrm{t}$ de ouro contido.

\subsection{Circuito Industrial}

O fluxograma de processo está ilustrado na Figura 1. A descrição detalhada do processo pode ser encontrada em Bergerman (2009) e em Nankran et al (2007). A descrição abaixo referese ao circuito de flotação e remoagem, foco deste trabalho.
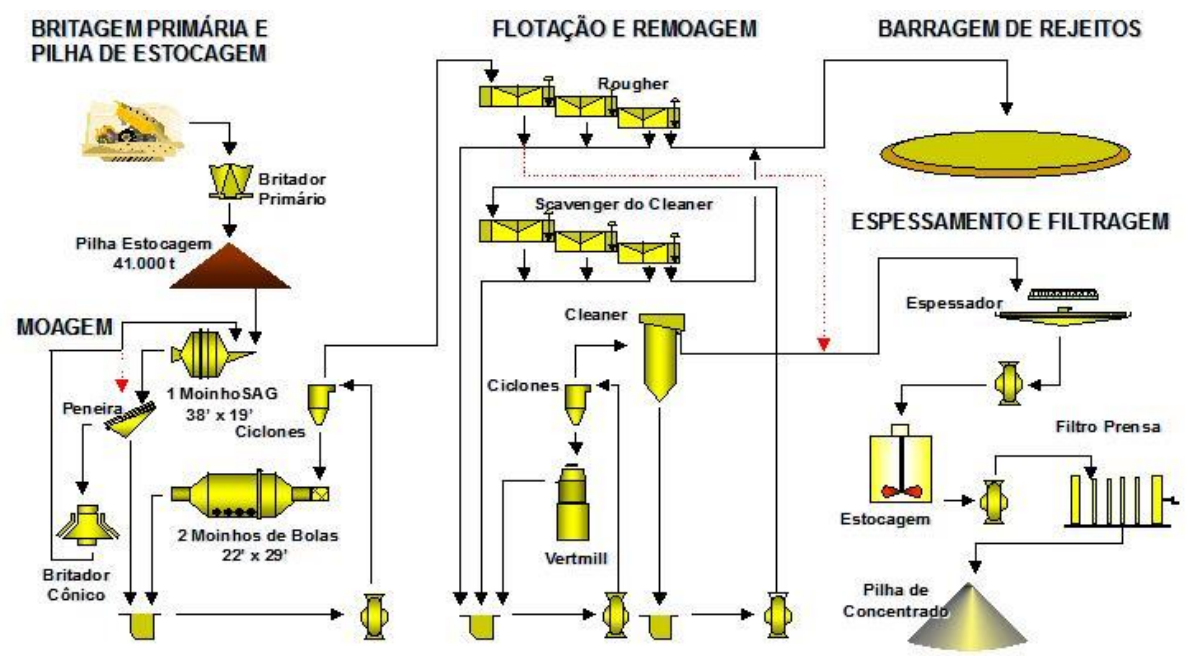

Figura 1 - Fluxograma de processo da usina do Sossego (NANKRAN et al, 2007)

O overflow das ciclonagens da moagem de bolas constitui a alimentação da etapa rougher de flotação, caracterizada por um $\mathrm{P}_{80}$ de aproximadamente $0,210 \mathrm{~mm}$ e $35 \%$ de sólidos. A flotação é composta por três etapas: rougher, cleaner escavenger.

A etapa de flotação rougher consiste em duas linhas paralelas e independentes, constituídas por sete células tanque cada, com capacidade de $160 \mathrm{~m}^{3}$ e aeração forçada.

O rejeito rougher corresponde a $95 \%$ do rejeito final, sendo direcionado à barragem com vazão média de $1.740 \mathrm{t} / \mathrm{h}$. Ressalta-se ainda que o pH médio da alimentação é de aproximadamente 8,7. As características dos produtos rougher estão relacionadas na Tabela 1. 
Tabela 1 - Valores típicos dos produtos da etapa rougher

\begin{tabular}{l|c|c}
\hline Produto & Teor de Cobre (\%) & Sólidos (\%) \\
\hline Alimentação & $0,9-1,3$ & $33-38$ \\
Concentrado & $13-17$ & $20-25$ \\
Rejeito & $0,05-0,07$ & $33-38$ \\
\hline
\end{tabular}

O concentrado rougher possui teor de cobre variando entre 13 a $17 \%$, com recuperação metalúrgica entre 94 e $96 \%$. Vale reportar que o circuito rougher dispõe da alternativa dos concentrados rougher das três primeiras células de cada linha alimentando diretamente o espessador ou a flotação cleaner (sem passar pela remoagem). Com esta alternativapromove-se mais ainda a recuperação global na flotação.

Os concentrados rougher e scavenger do cleaner alimentam, por meio de bombas de velocidade variável, duas baterias de 7 ciclones de 15". O underflow da ciclonagem é direcionado, por gravidade, para dois moinhos verticais modelo VTM-1500, que operam em circuito fechado com a ciclonagem (NANKRAN et al., 2007). Os corpos moedores são de $20 \mathrm{~mm}$. A alimentação dos ciclones (recalque da bomba) é dotada de medidor de pressão, vazão e densidade, permitindo assim um ótimo controle da vazão de alimentação dos ciclones e consequentemente dos moinhos verticais.

A fim de flexibilizar este circuito, foi instalada uma alternativa operacional para os casos de manutenção de um dos moinhos verticais. Pode-se encaminhar o material proveniente do circuito rougher e scavenger do cleaner diretamente ao circuito cleaner. Esta alternativa pode engrossar um pouco a alimentação da etapa cleaner, porém não a ponto de prejudicar a operação da mesma. $O$ teor do concentrado também pode sofrer ligeira queda, mas que não impacta significativamente a qualidade, tendo em vista os curtos períodos de tempo em que este circuito é acionado.

O produto da remoagem (overflow dos ciclones), com $\mathrm{P}_{80}$ da ordem de $44 \mu \mathrm{m}$, é submetido à flotação cleaner em seis colunas de flotação de 4,27 m de diâmetro e $14 \mathrm{~m}$ de altura, gerando um concentrado com aproximadamente 2,8 a $3 \%$ de cobre.

$O$ rejeito da etapa cleaner, com teor aproximado de $4 \%$ de cobre, alimenta a etapa scavenger do cleaner, composta de 6 células de flotação tipo tanque com volume de $160 \mathrm{~m}^{3}$.

O circuito de flotação possui uma recuperação mássica que está entre 3,5 a 4,0\%, com recuperação de cobre em torno de $93 \%$, que chega a picos de $98 \%$, em situações ótimas. 0 concentrado final é bombeado para o espessador e posteriormente filtrado.

\subsection{Dimensionamento do Circuito}

Para o dimensionamento do circuito de remoagem da usina do Sossego, foram realizados 4 ensaios de jarro pela empresa Metso, em seu laboratório de York, EUA. Os ensaios foram realizados em jarro de metal seguindo procedimentos internos da Metso. As análises granulométricas foram realizadas utilizando-se peneiras para a alimentação e o analisador de partículas Malvern Mastersizer 2000 para os produtos. Os resultados dos ensaios estão ilustrados na Tabela 2. 
Tabela 2 - Resultados dos ensaios de jarro

\begin{tabular}{c|c|c|c|c|c}
\hline \multirow{2}{*}{ Tempo (min) } & Consumo específico & \multicolumn{4}{|c}{ Valor do P80 $(\mu \mathrm{m})$} \\
\cline { 3 - 5 } & de energia $(\mathrm{kWh} / \mathrm{t})$ & Amostra A & Amostra B & Amostra C & Amostra D \\
\hline 0 & 0 & 162,6 & 185,3 & 174,2 & 116,4 \\
\hline 10 & 3,3 & 79,8 & 105,0 & 65,3 & 65,4 \\
\hline 15 & 4,9 & 60,6 & 72,4 & & 49,6 \\
\hline 20 & 6,6 & 53,9 & 61,2 & 55,1 & 44,0 \\
\hline 25 & 8,3 & 50,1 & 54,2 & & 41,1 \\
\hline 30 & 9,9 & 43,4 & 46,3 & 46,9 & \\
\hline 35 & 11,5 & 40,7 & 40,5 & & \\
\hline 40 & 13,2 & 36,7 & 36,6 & 37,7 & \\
\hline
\end{tabular}

Baseado nos resultados dos ensaios de jarro e na experiência do fornecedor, um consumo específico de 15,5 kWh/t foi considerado para efeitos de projeto. A decisão de se selecionar o moinho vertical para esta aplicação se baseou em experiências industriais em operações semelhantes, nas quais reduções de consumo de energia em relação ao moinho de bolas de carga cadente para remoagem da ordem de 30 a 40\% (KNORR e ALLEN, 2010; MENACHO e REYES, 1987; PENA, 1990). A literatura também reporta ganhos de 30 a $50 \%$ com redução do consumo de corpos moedores e revestimentos (PENA, 1990). Nappier-Munn et al (1999) destaca que os moinhos de bolas de carga cadente são limitados em termos da energia que eles podem transmitir a carga, o que pode explicar a evolução dos moinhos de carga agitada, como os moinhos verticais, para moagens fina (15-40 $\mu \mathrm{m}$ ) e ultrafina (abaixo de $15 \mu \mathrm{m}$ ).

\section{MATERIAIS E MÉTODOS}

Desde o início da operação da usina do Sossego, uma rotina de amostragens industriais foi estabelecida no circuito de remoagem, de forma a avaliar a operação do mesmo. A Figura 2 ilustra os pontos de amostragem do circuito. Os pontos 1 e 2 correspondem aos fluxos de concentrados rougher e scavenger do cleaner, o ponto 3 o underflow da classificação, o ponto 4 a descarga do moinho vertical e o ponto 5 o overflow da classificação.

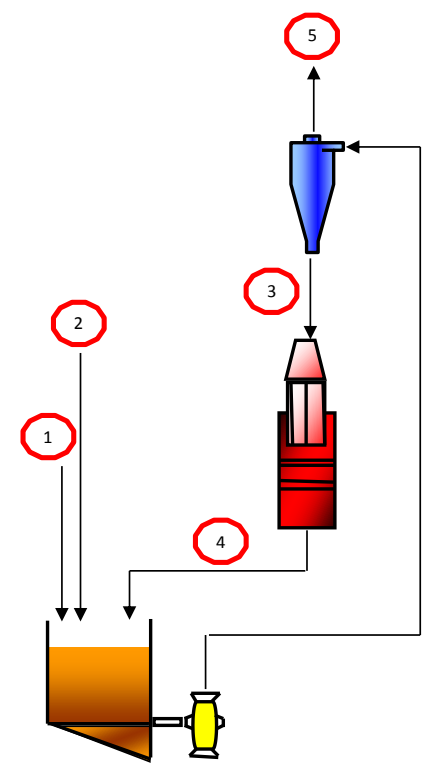

Figura 2 - Pontos de amostragem do circuito de remoagem do Sossego 
O circuito possui um medidor de vazão e densidade na linha de alimentação da classificação. O moinho é equipado com um medidor de corrente. Com estas informações e a porcentagem de sólidos e distribuição granulométrica dos 5 pontos ilustrados acima, é possível calcular a partição do ciclone e os seguintes índices para avaliação do consumo de energia do circuito.

- Índice de trabalho de Bond operacional (Wlop) - como demonstrado por Rowland e Kjos (1978), o índice de trabalho de Bond pode ser utilizado como um indicador do desempenho da operação industrial, baseado no Wlop. O Wlop pode ser calculado com base no $F_{80}$ e $P_{80}$ do circuito, massa nova de alimentação e potência efetivamente consumida pelo moinho. Neste trabalho, não foram aplicados os fatores de correção normalmente utilizados por Rowland e Kjos (1978), tendo em vista que os mesmos não foram determinados para os moinhos verticais.

- Consumo específico de energia ( $P / Q$ ) - trata-se da potência efetivamente consumida pelo moinho dividida pela massa nossa alimentada ao circuito.

\section{RESULTADOS E DISCUSSÕES}

A tabela 3 e a Figura 3 ilustram os resultados das amostragens realizadas desde 2006, com seus respectivos Wlop e consumo específico de energia. São relacionados também os resultados dos ensaios de jarro realizados em escala laboratorial para a amostra $D$, que se mostrou com uma granulometria de alimentação mais próxima a observada industrialmente.

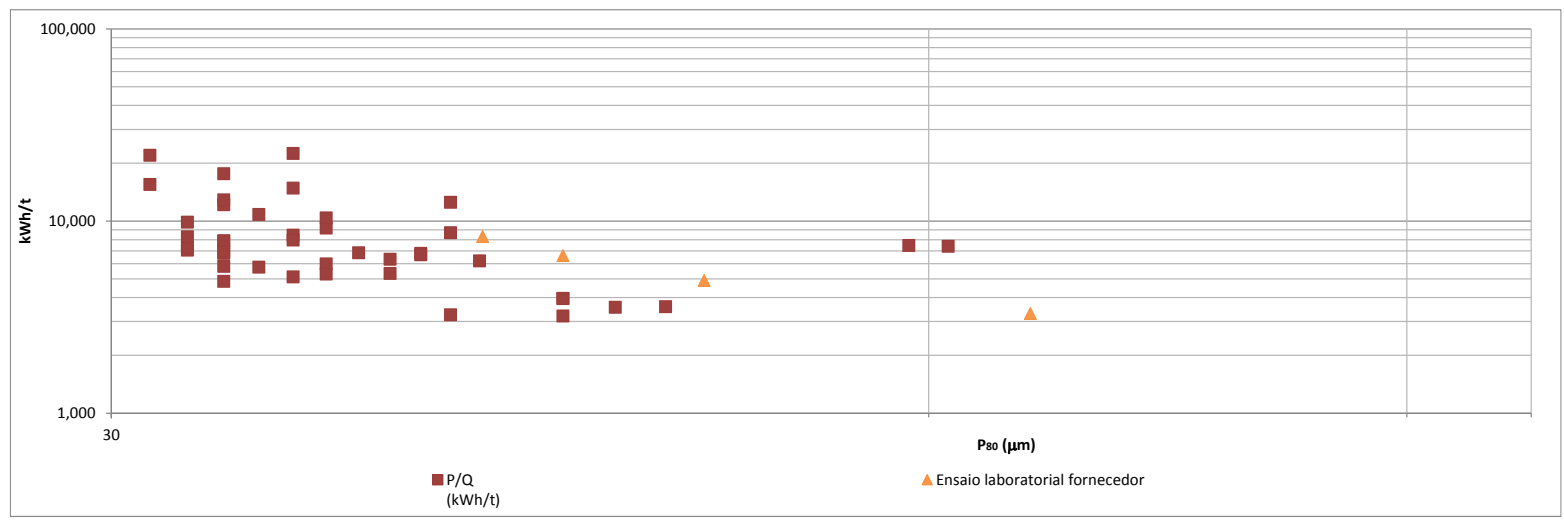

Figura 3 - Resultados de amostragens no circuito industrial do Sossego desde 2006

Ao observar os dados da Tabela 3 e da Figura 3, pode-se afirmar os resultados industriais do Sossego estão próximos aos valores de projeto. Isso valida que o circuito foi corretamente dimensionado, usando a metodologia proposta pelo fornecedor do equipamento. Estes resultados confirmam as observações de campo dos operadores da usina, já que o circuito de remoagem nunca foi um limitante para a operação da flotação. 
Tabela 3 - Resultados de amostragens no circuito industrial do Sossego desde 2006

\begin{tabular}{|c|c|c|c|c|c|c|c|c|}
\hline Data & $\begin{array}{l}\text { Potência } \\
\text { (kW) }\end{array}$ & $\begin{array}{c}\text { Alimentação } \\
\text { nova } \\
(\mathrm{t} / \mathrm{h})\end{array}$ & $\begin{array}{c}\mathrm{F} 80 \\
(\mathrm{~mm})\end{array}$ & $\begin{array}{c}\text { P80 } \\
(\mathrm{mm})\end{array}$ & $\mathrm{RR}$ & $\begin{array}{l}\text { Wiop } \\
(k W h / t)\end{array}$ & $\begin{array}{c}P / Q \\
(k W h / t)\end{array}$ & Moinho em operação \\
\hline Jar test Metso - D & & & 116 & 44 & 2,6 & 11,4 & 6,6 & \\
\hline Nominal & 1940 & 167 & 210 & 44 & 4,8 & 14,3 & 11,7 & \\
\hline Projeto & 2160 & 173 & 210 & 44 & 4,8 & 15,3 & 12,5 & \\
\hline $19 / 04 / 2006$ & 600 & 89 & 95 & 39 & 2,4 & 11,8 & 6,8 & MO01 \\
\hline $19 / 04 / 2006$ & 584 & 101 & 88 & 34 & 2,6 & 8,9 & 5,8 & MO02 \\
\hline $20 / 06 / 2006$ & 578 & 119 & 64 & 33 & 1,9 & 10,0 & 4,9 & MO02 \\
\hline $16 / 08 / 2006$ & 584 & 27 & 59 & 31 & 1,9 & 44,0 & 22,0 & MO01 \\
\hline $16 / 08 / 2006$ & 572 & 25 & 58 & 35 & 1,6 & 60,3 & 22,5 & MO02 \\
\hline $18 / 09 / 2006$ & 578 & 146 & 106 & 44 & 2,4 & 7,4 & 4,0 & MO01 \\
\hline $18 / 09 / 2006$ & 578 & 93 & 78 & 41 & 1,9 & 14,6 & 6,2 & MO01 \\
\hline $30 / 10 / 2006$ & 578 & 46 & 107 & 40 & 2,7 & 20,3 & 12,5 & MO01 \\
\hline $30 / 10 / 2006$ & 584 & 163 & 98 & 48 & 2,0 & 8,2 & 3,6 & MO02 \\
\hline $28 / 11 / 2006$ & 578 & 163 & 68 & 46 & 1,5 & 13,5 & 3,6 & MO01 \\
\hline $28 / 11 / 2006$ & 584 & 180 & 97 & 40 & 2,4 & 5,7 & 3,2 & MO02 \\
\hline $23 / 02 / 2007$ & 533 & 34 & 56 & 31 & 1,8 & 33,4 & 15,5 & MO01 \\
\hline $23 / 02 / 2007$ & 572 & 86 & 88 & 39 & 2,3 & 12,5 & 6,7 & MO02 \\
\hline $11 / 03 / 2008$ & 758 & 87 & 88 & 40 & 2,2 & 17,0 & 8,7 & MO01 \\
\hline $11 / 11 / 2008$ & 662 & 129 & 54 & 35 & 1,5 & 15,8 & 5,1 & MO01 \\
\hline $21 / 11 / 2008$ & 718 & 135 & 111 & 38 & 2,9 & 7,9 & 5,3 & MO02 \\
\hline $24 / 04 / 2009$ & 1431 & 81 & 36 & 33 & 1,1 & 254,8 & 17,6 & MO01 \\
\hline $15 / 06 / 2009$ & 673 & 45 & 142 & 35 & 4,1 & 17,4 & 14,8 & MO02 \\
\hline $24 / 07 / 2009$ & 701 & 83 & 128 & 35 & 3,7 & 10,5 & 8,4 & MO01 \\
\hline $23 / 11 / 2009$ & 808 & 139 & 87 & 33 & 2,6 & 8,6 & 5,8 & MO01 \\
\hline $30 / 07 / 2010$ & 735 & 61 & 91 & 33 & 2,8 & 17,6 & 12,2 & MO02 \\
\hline $13 / 08 / 2010$ & 791 & 200 & 102 & 44 & 2,3 & 7,7 & 4,0 & MO01 \\
\hline $13 / 08 / 2010$ & 612 & 191 & 92 & 44 & 2,1 & 6,9 & 3,2 & MO02 \\
\hline 07/10/2010 & 797 & 108 & 149 & 61 & 2,4 & 16,0 & 7,4 & MO01 \\
\hline $17 / 11 / 2010$ & 797 & 133 & 117 & 36 & 3,2 & 8,1 & 6,0 & MO01 \\
\hline $15 / 12 / 2010$ & 741 & 140 & 123 & 36 & 3,4 & 6,9 & 5,3 & MO01 \\
\hline $05 / 04 / 2011$ & 791 & 116 & 85 & 37 & 2,3 & 12,3 & 6,8 & MO01 \\
\hline $05 / 04 / 2011$ & 836 & 132 & 97 & 38 & 2,5 & 10,4 & 6,3 & MO02 \\
\hline $27 / 04 / 2011$ & 791 & 100 & 85 & 33 & 2,6 & 12,0 & 7,9 & MO01 \\
\hline $24 / 07 / 2011$ & 701 & 88 & 216 & 35 & 6,2 & 7,9 & 8,0 & MO01 \\
\hline $17 / 10 / 2011$ & 791 & 117 & 127 & 33 & 3,8 & 8,0 & 6,8 & MO01 \\
\hline $13 / 12 / 2011$ & 774 & 320 & 69 & 32 & 2,2 & 12,5 & 7,1 & MO01 \\
\hline $14 / 12 / 2011$ & 774 & 163 & 53 & 32 & 1,6 & 18,8 & 7,3 & MO01 \\
\hline $14 / 12 / 2011$ & 735 & 165 & 53 & 33 & 1,6 & 21,6 & 7,9 & MO02 \\
\hline $15 / 12 / 2011$ & 774 & 159 & 71 & 34 & 2,1 & 20,6 & 10,8 & MO01 \\
\hline $15 / 12 / 2011$ & 730 & 176 & 76 & 36 & 2,1 & 17,8 & 9,2 & MOO2 \\
\hline $26 / 01 / 2012$ & 713 & 157 & 35 & 32 & 1,1 & 113,5 & 9,9 & MO02 \\
\hline $19 / 04 / 2012$ & 819 & 141 & 108 & 32 & 3,4 & 10,3 & 8,3 & MO02 \\
\hline $16 / 08 / 2012$ & 735 & 141 & 55 & 36 & 1,5 & 32,5 & 10,4 & MO02 \\
\hline $12 / 09 / 2012$ & 769 & 273 & 174 & 59 & 2,9 & 13,7 & 7,5 & MO02 \\
\hline $16 / 10 / 2012$ & 769 & 233 & 54 & 33 & 1,6 & 18,4 & 6,9 & MO02 \\
\hline $23 / 11 / 2012$ & 842 & 167 & 133 & 33 & 4,0 & 14,8 & 12,9 & MO02 \\
\hline
\end{tabular}

Uma particularidade interessante a se destacar é em relação as vazões mássicas observadas na alimentação do circuito, significativamente menores que as de projeto. Esse número se explica pela variação do teor de alimentação da usina, em muitos momentos menor que o utilizado para o dimensionamento do circuito, assim como do uso das alternativas operacionais citadas anteriormente, de direcionamento de parte do concentrado da etapa rougher diretamente ao espessador, aliviando assim o circuito de remoagem. 
Outro ponto que chamou a atenção foi a granulometria de alimentação da remoagem, também mais fina que a projetada em grande parte das observações. Este ponto mereceu uma avaliação em detalhes, já que a alimentação da flotação estava dentro dos valores de projeto. Uma comparação posterior mostrou que o concentrado rougher é mais fino que a alimentação rougher. Esse é um ponto importante a se considerar em novos projetos. As origens desta diferença são partes de um estudo com mais detalhes, que se encontram em andamento.

Em termos de manutenção, o moinho vertical do Sossego também possui um consumo muito baixo de revestimentos. Apenas os revestimentos da rosca interna são trocados aproximadamente uma vez ao ano. $O$ consumo de corpos moedores também é baixo, da ordem de $20 \mathrm{~g} / \mathrm{t}$.

Baseado nestes resultados, tornou-se rotina na Vale utilização dos moinhos verticais em seus novos projetos, como o Salobo (GODOY et al., 2010).

No momento, direcionam-se esforços para o desenvolvimento de um ensaio simplificado de laboratório para avaliar as características do minério em termos de consumo de energia, usando amostras de pequenas massas, cerca de 1,0 kg e metodologia adaptada da proposta Donda (2003). Este ensaio será importante para que a Vale possa avaliar a variabilidade do minério, sem depender de ensaios realizados em laboratórios fora do país e realizados com procedimentos proprietários do fabricante do equipamento. Além disso, irá apoiar o desenvolvimento de novos projetos, que poderão ser avaliados por meio de uma base de dados mais extensa, já que por consumir menor massa, poderá ser realizado em amostras de ensaios de variabilidade de flotação de escala laboratorial.

\section{CONCLUSÕES}

Este trabalho descreveu os critérios utilizados para projeto e os resultados operacionais e de processo dos moinhos verticais do Sossego em seus primeiros anos de operação. Os resultados operacionais indicam que o dimensionamento foi realizado corretamente, sendo que os valores obtidos industrialmente em termos de consumo de energia e granulometria são iguais ou menores que os previstos em projeto. O circuito nunca foi um entrave para a operação da flotação. Os custos de manutenção e de consumo de corpos moedores também exibiram valores baixos. Diante desses resultados, a Vale continua considerando a utilização de tais equipamentos em novos projetos, como o Salobo.

\section{AGRADECIMENTOS}

Os autores agradeceem a Vale pelo apoio no desenvolvimento deste estudo e permissão para publicar o trabalho e a toda a equipe de processo do Sossego pelo longo trabalho de campo para coleta de todas as informações. Os autores agradecem também a Fapemig e a Pró-reitoria de pesquisa e pós-graduação da Unifal-MG pelo apoio para a publicação deste trabalho.

\section{REFERENCIAS BIBLIOGRÁFICAS}

1. BeRGERMAN, M. B. Modelagem e simulação do circuito de moagem do Sossego. 2009. 207 p. 
Dissertação (Mestrado) - Escola Politécnica, Universidade de São Paulo, São Paulo, 2009.

2. DONDA, J. D. Um método para prever o consumo especifico de energia na (re)moagem de concentrados de minério de ferro em moinhos de bolas. 2003. Tese (Doutorado) - Escola de engenharia, Universidade Federal de Minas Gerais, Belo horizonte, 2003.

3. GODOY, M. L. S., BERGERMAN, M. G., GODOY, P. H., ROSA, M. A. N., 2010. Development of the Salobo Project, in Proceedings: CIM Conference and Exhibition 2010 (The Canadian Institute of Mining, Metallurgy and Petroleum: Vancouver).

4. KNORR, B. R., ALLEN, J., 2010. Selection criteria of stirred milling technology, in Proceedings COMMINUTION' 2010 (Minerals Engineering conference: Cape Town).

5. MENACHO, J. M.; REYES, J. M. El Molino de torre como alternative en la remolienda de concentrados de cobre. In: V Simposium sobre molienda, 1987, Vinã del Mar. Proceedings: Vinã del Mar: ARMCO, 1987.

6. NAPIER-MUNN, T J et al., 1999. Mineral comminution circuits: their operation and optimization. Indooroopilly: Julius Kruttschnitt Mineral Research Centre/University of Queensland, 1999 (JKMRC Monograph Series in Mining and Mineral Processing).

7. PENA, F. Update on Vertimills for the mining industry. In: VI Simposium sobre molienda, 1990, Vinã del Mar. Proceedings: Vinã del Mar: ARMCO, 1990.

8. ROWLAND, C. A., KJOS, D. M., 1978. Rod and ball mills, in Mineral Processing Plant Design (ed. AIME), pp. $239-278$ (The American Institute of Mining, Metallurgical, and Petroleum : Englewood). 